\title{
Covalent organic polymer functionalization of activated carbon surfaces through acyl chloride for environmental clean-up
}

Mines, Paul D.; Thirion, Damien ; Uthuppu, Basil; Hwang, Yuhoon; Jakobsen, Mogens Havsteen; Andersen, Henrik Rasmus; Yavuz, Cafer T.

\section{Published in:}

Chemical Engineering Journal

Link to article, DOI:

10.1016/j.cej.2016.10.085

Publication date:

2017

Document Version

Peer reviewed version

Link back to DTU Orbit

Citation (APA):

Mines, P. D., Thirion, D., Uthuppu, B., Hwang, Y., Jakobsen, M. H., Andersen, H. R., \& Yavuz, C. T. (2017). Covalent organic polymer functionalization of activated carbon surfaces through acyl chloride for environmental clean-up. Chemical Engineering Journal, 309, 766-771. https://doi.org/10.1016/j.cej.2016.10.085

\section{General rights}

Copyright and moral rights for the publications made accessible in the public portal are retained by the authors and/or other copyright owners and it is a condition of accessing publications that users recognise and abide by the legal requirements associated with these rights.

- Users may download and print one copy of any publication from the public portal for the purpose of private study or research.

- You may not further distribute the material or use it for any profit-making activity or commercial gain

- You may freely distribute the URL identifying the publication in the public portal 


\title{
Covalent organic polymer functionalization of activated carbon surfaces through acyl chloride for environmental clean-up
}

\author{
Paul D. Mines, ${ }^{\mathrm{a}, \mathrm{b},{ }^{*}}$ Damien Thirion, ${ }^{\mathrm{b}}$ Basil Uthuppu, ${ }^{\mathrm{c}}$ Yuhoon Hwang, ${ }^{\mathrm{a}, \mathrm{d}}$ Mogens H. Jakobsen, ${ }^{\mathrm{c}}$ Henrik R. \\ Andersen, ${ }^{a}$ and Cafer T. Yavuz ${ }^{\text {b,* }}$ \\ ${ }^{a}$ Department of Environmental Engineering, Technical University of Denmark, Miljøvej. B113, DK-2800 \\ Kongens Lyngby, Denmark \\ ${ }^{\mathrm{b}}$ Graduate School of EEWS, Korea Advanced Institute of Science and Technology, 291 Daehak-ro, Yuseong-gu, \\ Daejeon 34141, Republic of Korea \\ ${ }^{c}$ Department of Micro- and Nanotechnology, Technical University of Denmark, Ørsteds Plads, B345B, DK- \\ 2800 Kongens Lyngby, Denmark \\ d Department of Environmental Engineering, Seoul National University of Science and Technology, 232 \\ Gongneung-ro, Nowon-gu, Seoul 01811, Republic of Korea
}

\begin{abstract}
Nanoporous networks of covalent organic polymers (COPs) are successfully grafted on the surfaces of activated carbons, through a series of surface modification techniques, including acyl chloride formation by thionyl chloride. Hybrid composites of activated carbon functionalized with COPs exhibit a core-shell formation of COP material grafted to the outer layers of activated carbon. This general method brings features of both COPs and porous carbons together for target-specific environmental remediation applications, which was corroborated with successful adsorption tests for organic dyes and metals.
\end{abstract}

Keywords: Activated carbon, Covalent organic polymers, Water treatment, Acyl chloride, Surface grafting 


\section{Introduction}

As water scarcity around the globe continues to deepen and water treatment becomes increasingly more crucial for the health and development of societies on every corner of the planet [1], the necessity for new, effective, and affordable treatment materials also becomes imperative. Cheap and efficient technologies employing novel materials are emerging across a wide range of disciplines, from nanomaterials [2-4], to biomimetic membranes [5,6], to modifications on classic technologies like activated carbon [7-10]. To date, the standard cheap adsorptive material in water treatment remains to be activated carbon. Activated carbon is, and has been for decades, actively employed in the adsorption of many common contaminants, such as pesticides [11], pharmaceuticals and endocrine disruptors [12], heavy metals [13], inorganic ions [14], among others. Although the versatility of activated carbon to adsorb a wide range of compounds is generally advantageous, it also becomes a limitation when there is a need for selectivity in treating a particular contaminant. Consequently, various modifications have been made on activated carbon to achieve specific advantages for numerous particular applications. These modifications have been treatment with acids, bases, external media impregnation, heat, microwaves, ozone, plasma, and bioadsorption $[15,16]$. Therefore, functionalization of this traditional material with novel compounds can expand its use to an even wider range of pollutants, targeting specific contaminated water sources. One of these novel materials is that termed covalent organic polymers (COPs). The benefit of these polymers is that they are extremely stable in many harsh conditions (e.g. high temperatures and boiling water) [17], and have already been proven efficient for environmental applications, such as gas capture [17-19], solvent uptake [20], and groundwater remediation [21].

However, full-scale applications of these COPs for environmental remediation have yet remained elusive. Efficient purification of large volumes of water or air requires that the contaminated stream can be drawn through a column containing highly functional materials, on the order of millimeters in size; in order to achieve 
economical synergy between contaminant/column contact time and energy demand. To date, COPs are nano- to micrometer in scale $[17,18,21,22]$ or in the form of macroscale solidified gel-like materials [20]. Columns built with particles of such a small size are rendered unpractical for typical remediation purposes, as they yield very high back pressures, as is the case with high performance liquid chromatography (HPLC) columns; and the energy to overcome this phenomenon would be too great for large-scale operation. Additionally, polymerizing the solidified gel-like polymers into millimeter-scale particles would still be limited in treatment efficacy due to slow intra-polymeric diffusion and insufficient contaminant contact time with the column material. Thus, supporting COPs on a cheap backbone material provides an ideal solution for creating a cost effective purification process.

Herein, we report the functionalization of COPs onto the surface of granular activated carbon (GAC); through a series of surface modification techniques, followed by the synthesis of a COP "shell" around the carbon granule. Activated carbon, established as one of the cheapest and most effective environmental remediation materials of all time [23], provides the optimal base material for the attachment of COPs. As it is large enough to be able to be used in a low energy demand packed-bed column [24] and has a compatible chemistry for the grafting of polymers to its surface. A first of its kind, activated carbon with a porous polymer functionalized shell provides a robust and re-generable material with the durability and versatility for a wide range of environmental applications. By developing a cheap functionalized activated carbon with a novel porous polymer matrix, it becomes possible to drastically increase the target contaminant range for water treatment, while still remaining affordable.

In this study, we successfully functionalized the surface of GAC with the porous matrix of COP material, specifically COP-19 [25]. Through an array of characterization techniques, it was confirmed that not only was the COP material present, but was in fact grafted to the surface of the carbon materials in a shell-like fashion. Given this successful functionalization (surface polymerization) method, it is now possible to develop a 
carbon/COP "library" of various different polymers attached to GAC. This library will aim at targeting specific environmental applications, depending on the polymer; from heavy metal up-take, to organics adsorption, to impregnation with reactive iron for contaminant degradation, to gas-capture and biogas purification operations. Subsequently, initial adsorption studies on an azo dye, cadmium, and iron prove feasibility for the developed material to be utilized in environmental applications.

\section{Materials and methods}

Acid oxidation of the carbon was performed as follows. $25 \mathrm{~g}$ of Samchun carbon (AC) was combined with 250 $\mathrm{mL}$ of concentrated nitric acid $\left(\mathrm{HNO}_{3}, 60 \%\right)$ at $100{ }^{\circ} \mathrm{C}$ under reflux for $24 \mathrm{~h}$. The carbon was washed thoroughly with copious amounts of MilliQ deionized water, until the rinsate reached a neutral $\mathrm{pH}$. This material (AC-Ox) was then dried in a vacuum oven for $12 \mathrm{~h}$ at $110^{\circ} \mathrm{C}$. Acyl chlorination of AC-Ox was performed as follows. 2.5 $\mathrm{g}$ of oxidized carbon (AC-Ox) was mixed with a 2:1 mixture of dichloromethane $\left(\mathrm{CH}_{2} \mathrm{Cl}_{2}\right), 100 \mathrm{~mL}$, and thionyl chloride $\left(\mathrm{SOCl}_{2}\right), 50 \mathrm{~mL}$, and refluxed for $24 \mathrm{~h}$ in a nitrogen atmosphere. The solvents were removed from the material via rotary evaporation to afford the acyl chloride modified carbon (AC-Thio). Melamine attachment to AC-Thio was performed as follows. Avoiding contact with air, approximately $2.5 \mathrm{~g}$ of acyl chloride modified particles (AC-Thio) were immediately treated with a solution of $375 \mathrm{mg}$ of melamine $\left(\mathrm{C}_{3} \mathrm{H}_{6} \mathrm{~N}_{6}\right)$ dissolved in 150 $\mathrm{mL}$ of dimethyl sulfoxide $\left(\left(\mathrm{CH}_{3}\right)_{2} \mathrm{SO}\right)$ and $2.5 \mathrm{~mL}$ of diisopropylethylamine (DIPEA) that had been sonicated until complete dissolution had taken place, and heated to $120^{\circ} \mathrm{C}$ for $24 \mathrm{~h}$ in a nitrogen atmosphere. After filtration, the particles of melamine attached carbon (AC-Mel) were washed thoroughly three times each with dimethyl sulfoxide, then MilliQ deionized water, and finally ethanol; then dried in a vacuum oven for $12 \mathrm{~h}$ at $110{ }^{\circ} \mathrm{C}$. Covalent organic polymer attachment to AC-Mel was performed as follows. $500 \mathrm{mg}$ of melamine and $800 \mathrm{mg}$ of terephthalaldehyde was combined with $150 \mathrm{~mL}$ of dimethyl sulfoxide, sonicated until complete dissolution took place, and placed into a flask with a nitrogen atmosphere. $1000 \mathrm{mg}$ of the melamine attached carbon particles (AC-Mel) were mixed into the solution, maintaining the nitrogen atmosphere. This mixture was 
stirred under reflux for $48 \mathrm{~h}$. After filtration, the particles of polymer attached carbon (AC-COP) were vigorously washed thoroughly three times each with dimethyl sulfoxide, then acetone, then MilliQ deionized water, and finally ethanol; then dried in a vacuum oven for $12 \mathrm{~h}$ at $110{ }^{\circ} \mathrm{C}$. Further information about the chemicals and equipment, and adsorption tests, can be found in the Supplementary material, section S1 and S2.

\section{Results and discussion}

Initial surface modification methods of the activated carbon were inspired from previously reported literature of surface modification using thionyl chloride targeted at graphene [26] and carbon black [27], as well as an aminofunctionalization method for carbon nanotubes [28]. Various types of GAC were tested for the ability to be effectively oxidized at the surface to introduce carboxylic groups, and it was discovered that the lack of impurities in a pristine GAC (i.e. Norit ${ }^{\circledR}$ activated carbon) made it difficult to break the bonds of the carbon and subsequently change the surface chemistry enough to allow for polymer grafting. Moreover, when using a GAC with too many impurities (i.e. Sigma activated charcoal), the surface area was too low and particle structure broke down too easily during modification. For mass loss, due to impurities on the carbons, determined by thermogravimetric analysis (TGA) of the different carbons, see Supplementary material, section S3. Consequently, a moderately pristine GAC (i.e. Samchun activated carbon) that still possesses a high surface area was found to be the optimal starting point for the reaction. Carbon was first oxidized with concentrated nitric acid $\left(\mathrm{HNO}_{3}, 60 \%\right)$ at $100{ }^{\circ} \mathrm{C}$ for $24 \mathrm{~h}(\mathrm{AC}-\mathrm{Ox})$. Once highly saturated with carboxylic groups, the carbon was treated with a 2:1 mixture of dichloromethane $\left(\mathrm{CH}_{2} \mathrm{Cl}_{2}\right)$ and thionyl chloride $\left(\mathrm{SOCl}_{2}\right)$ under reflux for $24 \mathrm{~h}(\mathrm{AC}$ Thio), converting the carboxylic groups to the extremely reactive acyl chloride substituent. After drying by rotary evaporation, the AC-Thio was used immediately in the next reaction step, without characterization, in order to avoid hydrolysis of acyl chloride by air or moisture. AC-Thio particles were then treated with a solution of melamine dissolved in dimethyl sulfoxide (DMSO) at $120{ }^{\circ} \mathrm{C}$ for $24 \mathrm{~h}$ (AC-Mel). At this point, melamine is grafted onto the surface of the acyl chloride containing carbon, by making amide bonds. Using the amine groups 
that melamine possesses, attachment of a covalent organic polymer (COP) shell around the GAC is thus possible. The final surface polymerization of attaching a COP matrix is employed by previously reported methods utilizing Schiff base chemistry with melamine and terephthaldehyde as the core monomers (AC-COP) $[22,25]$. The entire series of reaction schemes is identified in Figure 1. Due to the vigorous washing strategies after each phase of the grafting procedure, it can be assumed that any unattached monomers or polymers are no longer present (i.e. the high solubility of melamine in water and terephthaldehyde in ethanol will wash away any residuals), and therefore characterization data reflects only the carbon and grafted materials.

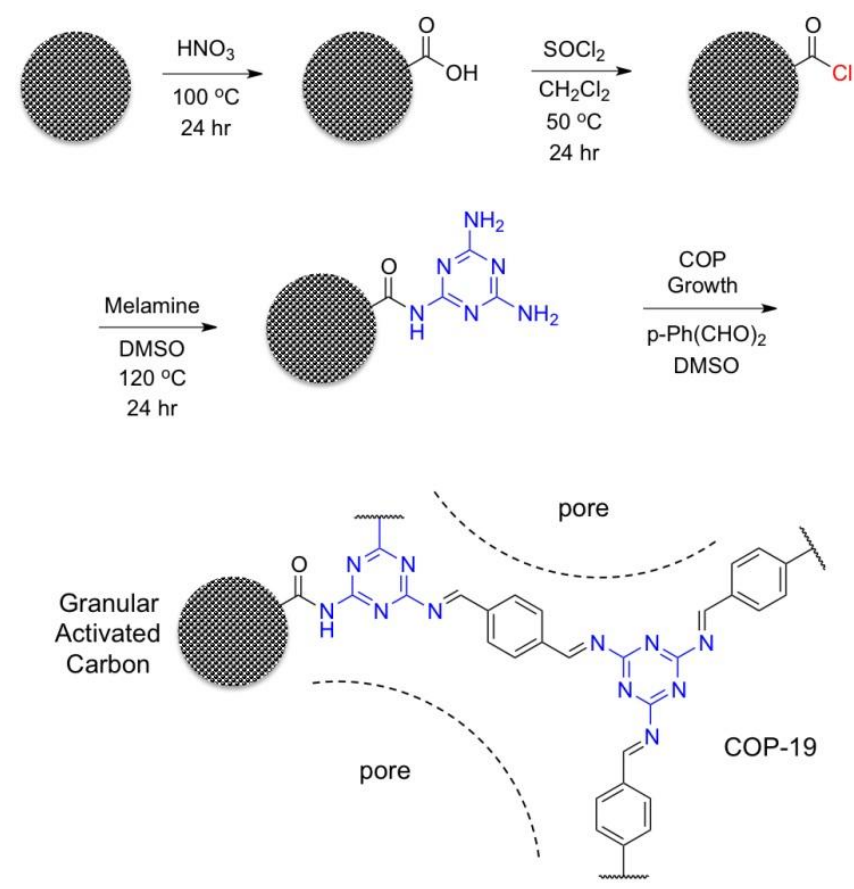

Fig. 1. Step-wise evolution of the COP attachment to the surface of activated carbon (illustrated as large grey object, not to scale).

Fourier transform infrared spectroscopy (FTIR) was carried out at each point in the series of reaction steps producing the carbon-COP composite (Figure 2A). As expected, the raw activated carbon exhibits no distinguishable peak pattern in the spectra, confirming the lack of any significant functional groups. Following 
the first reaction step with acid oxidation (AC-Ox), two major peaks appear at $1710 \mathrm{~cm}^{-1}$ and $1210 \mathrm{~cm}^{-1}$, which can be attributed to $\mathrm{C}=\mathrm{O}$ and $\mathrm{C}-\mathrm{O}$, respectively, of the stretching of carboxylic groups formed on the carbon surface. Another peak found at $1520 \mathrm{~cm}^{-1}$, correlates to nitro groups formed during the oxidation. After the reaction in the third step with the grafting of melamine (AC-Mel), the appearance of peaks at 1580 and $1000 \mathrm{~cm}^{-}$ ${ }^{1}$ correlate to $\mathrm{N}-\mathrm{H}$ and $\mathrm{C}-\mathrm{N}$, respectively. These stretching frequencies verify the existence of secondary amine groups stemming from the acyl chloride on the carbon surface. Ultimately, the final product (AC-COP) following the reaction in the fourth step with the COP attachment, yields a multitude of peaks at 1720, 1530, $1460,1340,1190,980,860$, and $800 \mathrm{~cm}^{-1}$. These peaks are identical to the peak pattern found in the pure COP19, confirming that there is now COP material contained within the sample matrix (Figure 2A, green dashed line).
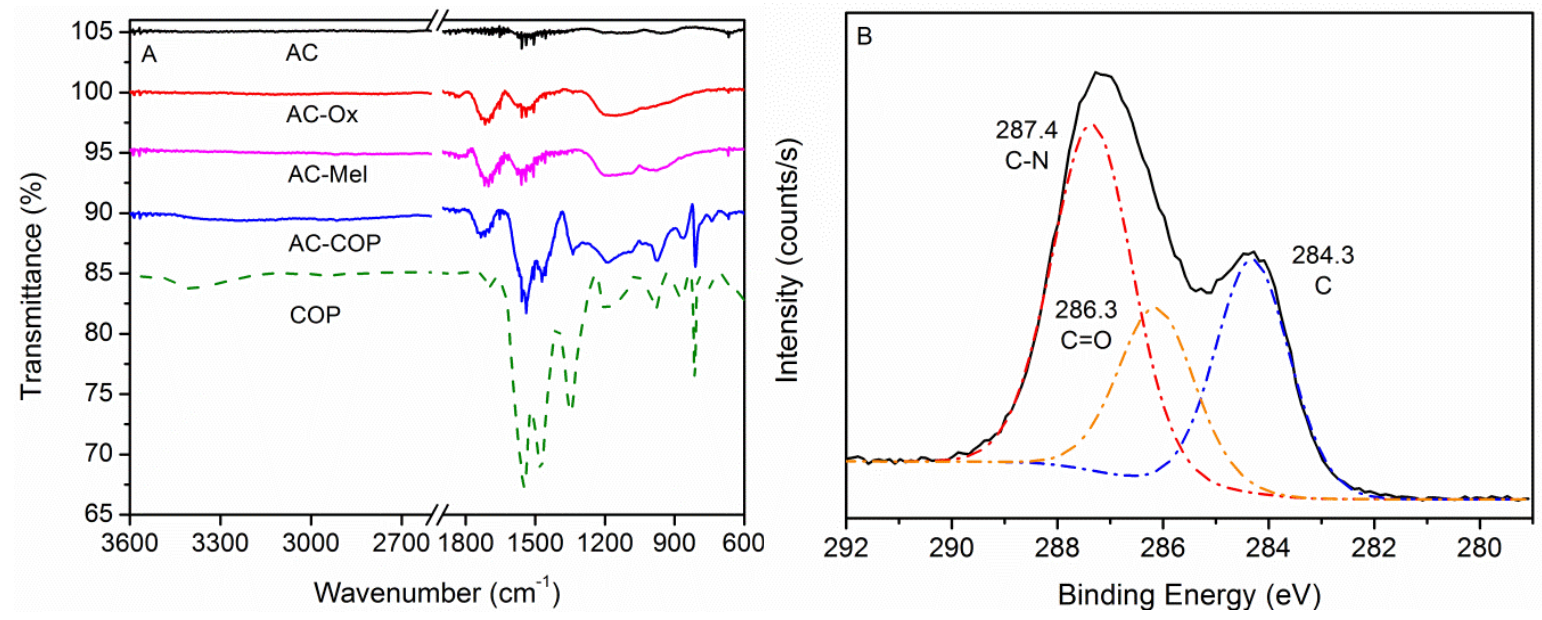

Fig 2. A) FTIR spectra of AC (black), AC-Ox (red), AC-Mel (magenta), AC-COP (blue), and COP-19 (green); XPS C1s scan of AC-COP.

When elemental analysis (EA) was performed on the carbon particles, there was a clear trend in the quantities of each element present that corresponded very well with the reaction scheme (Table 1A). Initially, as expected, the particles are primarily carbon, with some defects found mostly in the form of oxidation. Following acid 
oxidation with nitric acid (AC-Ox), the percentage of oxygen significantly increases; additionally, minor increases in hydrogen and nitrogen takes place. After treatment with thionyl chloride (AC-Thio), and with the attachment of melamine to the carbon surface (AC-Mel), the third step correspondingly sees a substantial increase in nitrogen and hydrogen, due to the added presence of amine groups and aromatic nitrogens; while concurrently seeing a significant decrease in oxygen, as a secondary amine substitutes for the acyl chloride. Furthermore, the COP attachment more than doubles the nitrogen content while lowering the oxygen and hydrogen slightly (AC-COP), matching well with the added presence of a polymer constructed from melamine and terephthaldehyde.

Table 1. Elemental concentrations of carbon particles at each stage of the modification reactions, determined by elemental analysis (A) and X-ray photoelectron spectroscopy (B).

\begin{tabular}{|c|c|c|c|c|c|}
\hline & $\mathrm{C}$ & $\mathrm{N}$ & $\mathrm{O}$ & $\mathrm{H}$ & Other \\
\hline \multicolumn{6}{|c|}{ A. Elemental analysis (\%) } \\
\hline $\mathrm{AC}$ & 82.40 & 0.58 & 6.50 & 1.67 & 8.85 \\
\hline AC-Ox & 55.43 & 2.16 & 33.40 & 2.04 & 6.97 \\
\hline AC-Mel & 61.90 & 7.57 & 19.48 & 4.23 & 6.82 \\
\hline AC-COP & 55.85 & 17.52 & 12.43 & 2.65 & 11.54 \\
\hline \multicolumn{6}{|c|}{ B. X-ray photoelectron spectroscopy (\%) } \\
\hline $\mathrm{AC}$ & 77.36 & 0.00 & 18.58 & --- & 4.06 \\
\hline AC-COP & 57.28 & 32.80 & 6.36 & --- & 3.56 \\
\hline
\end{tabular}

However, in order to verify that the spectral data from FTIR was indeed indicative of a polymer and its corresponding functional groups on the surface of the carbon, rather than entirely separate co-existing particles, X-ray photoelectron spectroscopy (XPS) was employed, which is limited to a penetration depth of the first 10 $\mathrm{nm}$ of a surface. Analyzing the elemental percentages with XPS reveals a clear indication that there is a polymer shell bound to the surface of the carbon, see Table 1B. The best indicator for this is the surface nitrogen, which is non-existent in the original carbon, and is nearly one-third of the total content when the polymer is grafted. Additionally, an in-depth look at the C1s scan of the XPS measurement yields three distinct peaks of C-N, C=O, 
and C (Figure 2B), whereas the original carbon contains only the bare carbon peak (see Supplementary material, section $\mathrm{S} 4)$.

Final confirmation of successful COP attachment to the carbon surface was verified using transmission electron microscopy (TEM). Imaging with TEM revealed large dark activated carbon particles, out of focus in the foreground, with a shell of interwoven porous chains of COP material bound to the outer edges, in focus in the background (Figure 3). Confirming expected polymer formation and construction, the morphology of the COP used in this study and observed surrounding the GAC closely resembled that of the COP used in a previous study immobilizing nanoscale zero-valent iron (nZVI) into the pores of the COP (see Supplementary material, section S5) [21]. In addition, when TEM imaging was performed on particles just before COP attachment (AC-Mel) with only melamine bound to the surface, there was a noticeable difference in the material at the edges of the activated carbon. This material of melamine monomers attached appeared as an amorphous and nonporous coating, rather than a shell of a porous polymer matrix (see Supplementary material, section S5).

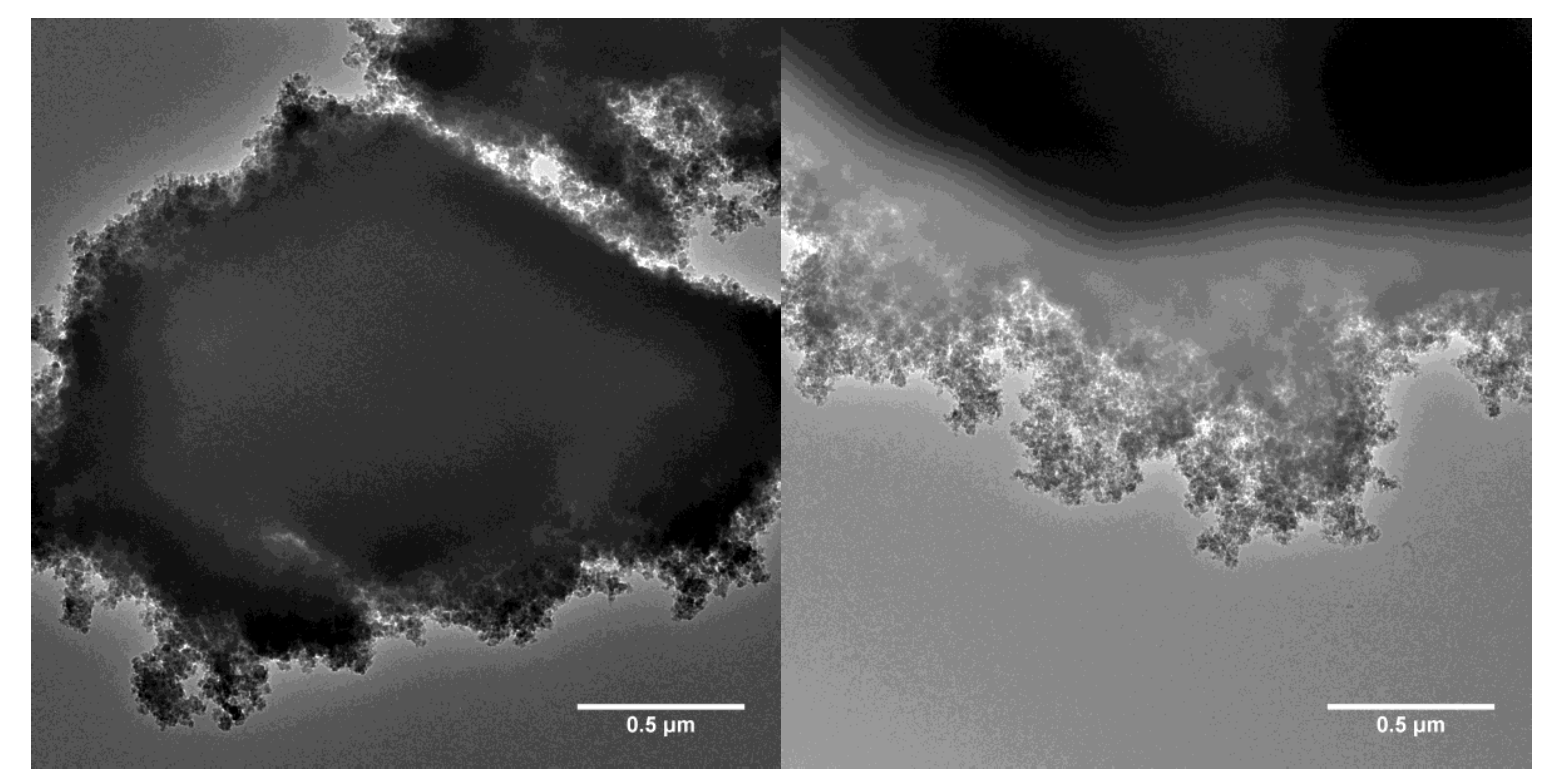

Fig. 3. TEM images of activated carbon particles with an attached shell of COP material. 
Porosity and textural characterization was performed on the materials, using nitrogen adsorption-desorption isotherms at 77K (Figure 4). Activated carbon shows a typical type I isotherm, due to its microporous nature, with a very narrow pore size distribution centered around $2 \mathrm{~nm}$ (Figure 4A, black line). For comparison, the bare COP possesses a type II isotherm with a significant amount of macropores and a broad range of pore sizes (from $2.6 \mathrm{~nm}$ to $100 \mathrm{~nm}$ ) (see Supplementary material, section S7). Not uncommon after oxidation of activated carbon $[29,30]$, the porosity of AC-Ox is significantly reduced, reflecting the high content of oxidized functionalities blocking the pores (Figure 4B, red line). Melamine attachment and successive COP growing leads to an increase in surface area of $75 \mathrm{~m}^{2} / \mathrm{g}$ and $338 \mathrm{~m}^{2} / \mathrm{g}$ for AC-Mel and AC-COP, respectively. The increased porosity can be explained through partially re-opening of the blocked pores after oxidation (small pores centered around $2.3 \mathrm{~nm}$ ), as well as newly generated larger pores from the COP structure itself (2.6 $\mathrm{nm}$ to $10 \mathrm{~nm}$ ) but with a broader pore size distribution, reflecting the hybrid nature of the AC-COP.
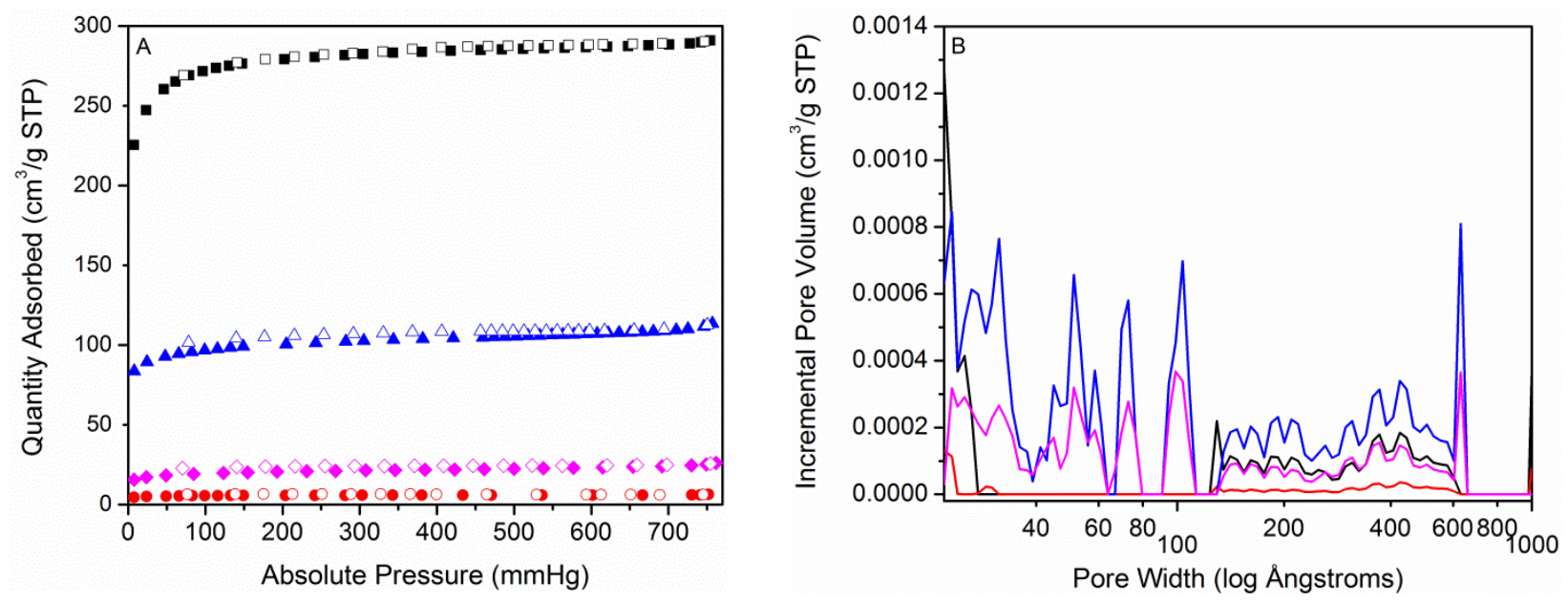

Fig. 4. A) $\mathrm{N}_{2}$ adsorption (filled)-desorption (empty) isotherms at $77 \mathrm{~K}$; B) Pore size/volume distribution by 2DNLDFT (slit pores, $\mathrm{N}_{2}$ carbon model) of AC (black), AC-Ox (red), AC-Mel (magenta), and AC-COP (blue).

Finally, in order to test the preliminary efficacy of the produced material for environmental clean-up and contaminant removal/adsorption, in separate batch tests, AC-COP was mixed with the azo dye naphthol blue 
black and cadmium. Iron was also tested, to assess possibility for future application of impregnating nZVI into AC-COP for subsequent contaminant degradation. For comparison, AC and COP-19 were also examined. After only 30 minutes, AC-COP and COP-19 exhibited a high affinity for adsorbing the azo dye, very similar to previously reported results using COP-19 [21]; while the AC did not, each adsorbing up to 23.6, 23.8, and 5.6 $\mathrm{mg} / \mathrm{g}$, respectively (Figure 5A). The high adsorption of an azo dye to the COP material has been exhibited before [21], and can best be explained by the phenomenon known as pi-pi stacking, which has been observed before between aromatic compounds [31-33]. After 72 hours, adsorption for cadmium was 5.6, 4.5, and 5.3 mg/g for AC-COP, COP-19, and AC, respectively (Figure 5B); similar results have been observed adsorbing cadmium with carbon nanotubes [34]. Interestingly, COP-19 exhibited the lowest cadmium adsorption, however, there was a synergistic effect observed between the AC and COP-19 in AC-COP, which adsorbed the highest amount of cadmium. After 72 hours, adsorption for iron was 8.7, 12.4, and 11.5 mg/g for AC-COP, COP-19, and AC, respectively (Figure 5C). Although, having lower adsorption numbers, AC-COP continued to increase adsorption of iron over a 3 day period, while AC and COP-19 saw a release of iron back into solution after 8 hours, indicating that AC-COP is permanently trapping iron, making it an ideal candidate of nZVI I impregnation for future studies on contaminant degradation. Ultimately, the rate constants of adsorption were determined using pseudo first-order kinetics [35]. The rate constants for azo dye adsorption were 0.0678, 0.1304, and $0.2211 \mathrm{~h}^{-1}$ for AC, COP-19, and AC-COP, respectively; the rate constants for cadmium adsorption were 0.0012, 0.0016, and $0.0011 \mathrm{~h}^{-1}$ for AC, COP-19, and AC-COP, respectively; and the rate constants for iron adsorption were $0.0203,0.0124$, and $0.0121 \mathrm{~h}^{-1}$ for AC, COP-19, and AC-COP, respectively. Preliminary adsorption batch tests indicate the ability of the developed material to be used effectively in environmental applications. 

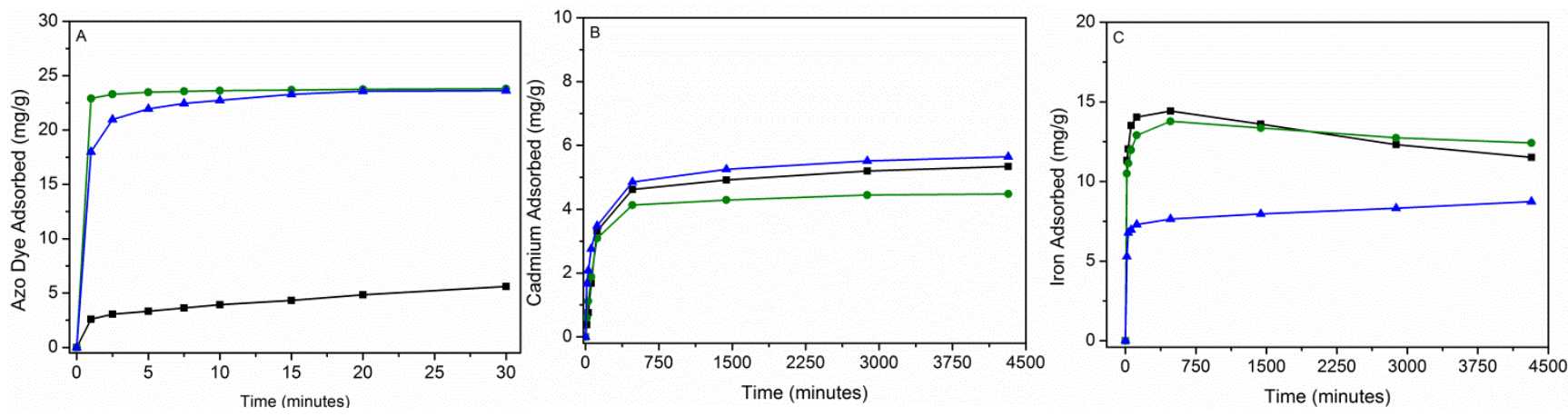

Fig. 5. Adsorption isotherms of A) azo dye naphthol blue black; B) cadmium; and C) iron adsorbed by AC-COP (blue), COP-19 (green) and AC (black). Material dosage: $0.5 \mathrm{~g} / \mathrm{L}$; initial pH: 6.5; $\mathrm{C}_{0}$-azo dye: $40 \mu \mathrm{M} ; \mathrm{C}_{0}$-Cd: 10 $\mathrm{mg} / \mathrm{L} ; \mathrm{C}_{0}-\mathrm{Fe}: 10 \mathrm{mg} / \mathrm{L}$.

\section{Conclusions}

In summary, although various types of activated carbons were tested, it was determined that very pristine or crude carbons are not adequate for surface modification. If the carbon is too pristine, then the surface oxidation becomes extremely difficult; and, if the carbon is too crude, then the structural integrity of the carbon becomes a concern. Therefore, a carbon with a moderate amount of defects that possesses a high surface area is an optimal material for polymer functionalization of GAC. Defects initially present in the carbon leads to ease of oxidation of the surface, creating functional carboxyl groups for further modification. These carboxyl groups are easily converted in to acyl chlorides via thionyl chloride treatment. From the acyl chloride, monomer attachment, in the form of melamine, is then possible. Monomers such as this can provide the building infrastructure for the construction of a polymeric network around the surface of the carbon. In this manner, we successfully created a covalent organic polymer shell around activated carbon granules, the first time this type of porous framework has been attached to the surface of activated carbon. From here, we expect to establish a "library" of surface modified polymer attached carbons for various environmental applications; ranging from heavy metal adsorption, 
to water contamination treatment, to $\mathrm{CO} 2$ adsorption, to biogas purification. Consequently, following the successful initial results of contaminant adsorption by AC-COP, the environmental applications are promising.

\section{Acknowledgements}

P.D.M., B.U., M.H.J., and H.R.A. acknowledge funding for this study from the Technical University of Denmark (DTU) through the KAIST-DTU Signature Project on Integrated Water Technology. Y.H. acknowledges funding for this study through a DFF-Individual Postdoctoral Grant from the Danish Council for Independent Research - Technology and Production Science (4005-00393B). D.T. and C.T. Yavuz acknowledge the financial support by Basic Science Research Program through the National Research Foundation of Korea (NRF), ICT \& Future Planning (2013R1A1A1012998), and IWT (NRF-2012-C1AAA001M1A2A2026588).

\section{References}

[1] U.N. Water, Coping with water scarcity: challenge of the twenty-first century. Report for World Water Day 2007, in: World Water Day, United Nations, Rome, Italy, 2007: pp. 1-29.

[2] C.T. Yavuz, J.T. Mayo, W.W. Yu, A. Prakash, J.C. Falkner, S. Yean, et al., Low-Field Magnetic Separation of Monodisperse Fe3O4 Nanocrystals, Science (80-. ). 314 (2006) 964-967.

[3] M.S. Diallo, C.J. Brinker, Nanotechnology for Sustainability: Environment, Water, Food, Minerals, and Climate, in: Nanotechnol. Res. Dir. Soc. Needs 2020 SE - 6, Springer Netherlands, 2011: pp. 221-259.

[4] Y. Hwang, Y.-C. Lee, P.D. Mines, Y.S. Huh, H.R. Andersen, Nanoscale zero-valent iron (nZVI) synthesis in a Mg-aminoclay solution exhibits increased stability and reactivity for reductive decontamination, Appl. Catal. B Environ. 147 (2014) 748-755.

[5] C.Y. Tang, Y. Zhao, R. Wang, C. Hélix-Nielsen, A.G. Fane, Desalination by biomimetic aquaporin 
membranes: Review of status and prospects, Desalination. 308 (2013) 34-40.

[6] Y. Zhao, C. Qiu, X. Li, A. Vararattanavech, W. Shen, J. Torres, et al., Synthesis of robust and highperformance aquaporin-based biomimetic membranes by interfacial polymerization-membrane preparation and RO performance characterization, J. Memb. Sci. 423-424 (2012) 422-428.

[7] S.A. Messele, O.S.G.P. Soares, J.J.M. Órfão, C. Bengoa, F. Stüber, A. Fortuny, et al., Effect of activated carbon surface chemistry on the activity of ZVI/AC catalysts for Fenton-like oxidation of phenol, Catal. Today. 240 (2015) 73-79.

[8] S.A. Messele, O.S.G.P. Soares, J.J.M. Órfão, F. Stüber, C. Bengoa, A. Fortuny, et al., Zero-valent iron supported on nitrogen-containing activated carbon for catalytic wet peroxide oxidation of phenol, Appl. Catal. B Environ. 154-155 (2014) 329-338.

[9] H.S. Niasar, H. Li, T.V.R. Kasanneni, M.B. Ray, C. (Charles) Xu, Surface amination of activated carbon and petroleum coke for the removal of naphthenic acids and treatment of oil sands process-affected water (OSPW), Chem. Eng. J. 293 (2016) 189-199.

[10] A. Rey, A.B. Hungria, C.J. Duran-Valle, M. Faraldos, A. Bahamonde, J.A. Casas, et al., On the optimization of activated carbon-supported iron catalysts in catalytic wet peroxide oxidation process, Appl. Catal. B Environ. 181 (2016) 249-259.

[11] K.Y. Foo, B.H. Hameed, Detoxification of pesticide waste via activated carbon adsorption process, J. Hazard. Mater. 175 (2010) 1-11.

[12] Z. Yu, S. Peldszus, P.M. Huck, Adsorption of Selected Pharmaceuticals and an Endocrine Disrupting Compound by Granular Activated Carbon. 1. Adsorption Capacity and Kinetics, Environ. Sci. Technol. 43 (2009) 1467-1473.

[13] M.O. Corapcioglu, C.P. Huang, The adsorption of heavy metals onto hydrous activated carbon, Water 
Res. 21 (1987) 1031-1044.

[14] A. Bhatnagar, M. Sillanpää, A review of emerging adsorbents for nitrate removal from water, Chem. Eng. J. 168 (2011) 493-504.

[15] C.Y. Yin, M.K. Aroua, W.M.A.W. Daud, Review of modifications of activated carbon for enhancing contaminant uptakes from aqueous solutions, Sep. Purif. Technol. 52 (2007) 403-415.

[16] A. Bhatnagar, W. Hogland, M. Marques, M. Sillanpää, An overview of the modification methods of activated carbon for its water treatment applications, Chem. Eng. J. 219 (2013) 499-511.

[17] H.A. Patel, F. Karadas, A. Canlier, J. Park, E. Deniz, Y. Jung, et al., High capacity carbon dioxide adsorption by inexpensive covalent organic polymers, J. Mater. Chem. 22 (2012) 8431-8437.

[18] H.A. Patel, F. Karadas, J. Byun, J. Park, E. Deniz, A. Canlier, et al., Highly Stable Nanoporous SulfurBridged Covalent Organic Polymers for Carbon Dioxide Removal, Adv. Funct. Mater. 23 (2013) 22702276.

[19] H.A. Patel, S.H. Je, J. Park, Y. Jung, A. Coskun, C.T. Yavuz, Directing the structural features of N(2)phobic nanoporous covalent organic polymers for $\mathrm{CO}(2)$ capture and separation., Chemistry. 20 (2014) $772-780$.

[20] H.A. Patel, M.S. Yavuz, C.T. Yavuz, Exceptional organic solvent uptake by disulfide-linked polymeric networks, RSC Adv. 4 (2014) 24320-24323.

[21] P.D. Mines, J. Byun, Y. Hwang, H.A. Patel, H.R. Andersen, C.T. Yavuz, Nanoporous networks as effective stabilisation matrices for nanoscale zero-valent iron and groundwater pollutant removal, J. Mater. Chem. A. 4 (2016) 632-639.

[22] M.G. Schwab, B. Fassbender, H.W. Spiess, A. Thomas, X. Feng, K. Müllen, Catalyst-free preparation of melamine-based microporous polymer networks through Schiff base chemistry., J. Am. Chem. Soc. 131 
(2009) 7216-7217.

[23] I.N. Najm, V.L. Snoeyink, B.W. Lykins, J.Q. Adams, Using Powdered Activated Carbon: A Critical Review, J. Am. Water Works Assoc. 83 (1991) 65-76.

[24] F. Meinel, A.S. Ruhl, A. Sperlich, F. Zietzschmann, M. Jekel, Pilot-Scale Investigation of Micropollutant Removal with Granular and Powdered Activated Carbon, Water, Air, Soil Pollut. 226 (2014) $1-10$.

H.A. Patel, C.T. Yavuz, Highly optimized CO2 capture by inexpensive nanoporous covalent organic polymers and their amine composites, Faraday Discuss. 183 (2015) 401-412.

[26] J.K. Wassei, K.C. Cha, V.C. Tung, Y. Yang, R.B. Kaner, The effects of thionyl chloride on the properties of graphene and graphene-carbon nanotube composites, J. Mater. Chem. 21 (2011) 3391-3396.

H. He, M. Zhong, D. Konkolewicz, K. Yacatto, T. Rappold, G. Sugar, et al., Carbon black functionalized with hyperbranched polymers: synthesis, characterization, and application in reversible CO2 capture, J. Mater. Chem. A. 1 (2013) 6810-6821.

[28] T. Ramanathan, F.T. Fisher, R.S. Ruoff, L.C. Brinson, Amino-Functionalized Carbon Nanotubes for Binding to Polymers and Biological Systems, Chem. Mater. 17 (2005) 1290-1295.

[29] M.A. Álvarez-Merino, V. López-Ramón, C. Moreno-Castilla, A study of the static and dynamic adsorption of $\mathrm{Zn}(\mathrm{II})$ ions on carbon materials from aqueous solutions, J. Colloid Interface Sci. 288 (2005) $335-341$.

[30] C. Moreno-Castilla, M.A. Ferro-Garcia, J.P. Joly, I. Bautista-Toledo, F. Carrasco-Marin, J. RiveraUtrilla, Activated Carbon Surface Modifications by Nitric Acid, Hydrogen Peroxide, and Ammonium Peroxydisulfate Treatments, Langmuir. 11 (1995) 4386-4392.

[31] B. Lippa, G. Pan, M. Corbett, C. Li, G.S. Kauffman, J. Pandit, et al., Synthesis and structure based 
optimization of novel Akt inhibitors, Bioorg. Med. Chem. Lett. 18 (2008) 3359-3363.

[32] Y. Morisaki, Y. Chujo, Synthesis of \&pi;-Stacked Polymers on the Basis of [2.2]Paracyclophane, Bull. Chem. Soc. Jpn. 82 (2009) 1070-1082.

[33] F.D. Lewis, P. Daublain, G.B. Delos Santos, W. Liu, A.M. Asatryan, S.A. Markarian, et al., Dynamics and Mechanism of Bridge-Dependent Charge Separation in Pyrenylurea-Nitrobenzene $\pi$-Stacked Protophanes, J. Am. Chem. Soc. 128 (2006) 4792-4801.

[34] Y.-H. Li, S. Wang, Z. Luan, J. Ding, C. Xu, D. Wu, Adsorption of cadmium(II) from aqueous solution by surface oxidized carbon nanotubes, Carbon N. Y. 41 (2003) 1057-1062.

[35] B.H. Hameed, A.T.M. Din, A.L. Ahmad, Adsorption of methylene blue onto bamboo-based activated carbon: Kinetics and equilibrium studies, J. Hazard. Mater. 141 (2007) 819-825. 